\title{
The Angular Distribution of Neutral Hydrogen Following Collisional Electron Detachment from $\mathrm{H}^{-}$
}

\author{
J. D. Moses \\ D. Holtkamp \\ J. D. King \\ P. W. Lisowski \\ J. E. Simmons
}


The Angular Distribution of Neutral Hydrogen Following collisional Electron Detachinent Frcin $\mathrm{H}^{-}$ by J. D. Moses, D. Holtkamp, J. D. King, P. W. Lisowski, and J.E. Simmons

\section{Abstract}

The angular distribution of neutral. hydrogen following electron detachment from $\mathrm{H}^{-}$has been measured for $\mathrm{H}^{-}$on nitrogen and hydrogen gas, both for neutral atoms left in the 2s state, and for all neutrals independent of the state of excitation. The results are essentially in agreement with calculations based on the Born approximation, although there is some disagreement in detail.

\section{Introduction}

The distribution in angle of neutral hydrogen following single collision electron detachment from $\mathrm{H}^{-}$should be well described by the Born approximation for incident energies in the MeV ragion, where the speed of the ion is greater than that of the atomic electrons. Angular distributions of the center of mass of the hydrogen atom and detachea electron have been calculated by Gillespie ${ }^{l}$ and by Lee and Chen. ${ }^{2}$ More recently Genoni and Wright ${ }^{3}$ and Franz, Wright and Genoni ${ }^{4}$ have emphasized that the motion of the stripped atom differs significantly from that of the center of mass and that the width of the distribution of atoms left in excited states is considerakly broader than that of atoms left in the ground state. 
Here we report measurements of the angular distribution of neutral hydrogen following stripping of $\mathrm{H}^{-}$on nitrogen and hydrogen gas. We present results both for atoms left in the 2s excited state and for neutral atoms independent of their state of excitation.

The apparatus used in these measurements will be described in Section II of this report, followed by a presentation of the experinental results and a simple analysis in section III. We present our conclusions in section IV.

\section{Apparatus}

\section{a. Overall considerations}

The angilar deflection of a hydrogen atom after collisional detachment of one electron from $\mathrm{H}^{-}$was estimated in the Born approximation $^{1-4}$ to be of the order of 10 microradians for hydrogen left in the grourd state. In corsequence, the $\mathrm{H}^{-}$ beam must be collimated to a few microradians, at least in one dimension. If the experiment is to be confined to a few meters overall length, a position-sensitive detector with precision of a few $\mu \mathrm{m}$ is needed. In addition, if excitedstate atoms are to be detected unambiguolisly, techniques are needed to tag these atoms. A schematic view of the apparatus we have developed for this measurement is shown in Fig. 1. The $\mathrm{H}^{-}$beam from the vertical Van de Graaff accelerator at the Los Alamos Ion Beam Facility passes through defining slits, where it is shaped to roughly $2 \mathrm{~mm}$ high by $1 / 2 \mathrm{~mm}$ wide. After 


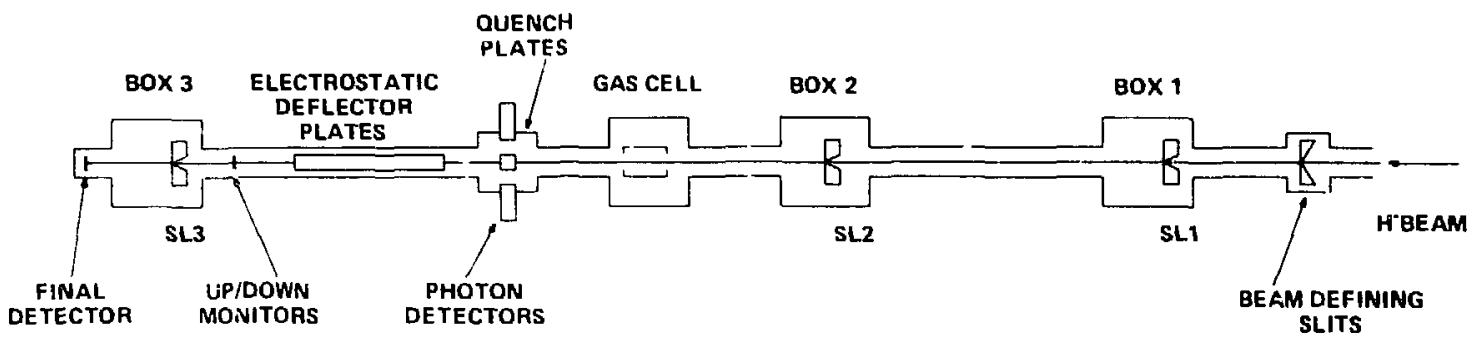

LONGITUDINAL SCALE (CM)

100

Fig. 1. Plan view of the experimental apparatus; scale is maintained in the beam direction.

this, two sets of precision-machined slits collimate the beam to a few microradians divergence in the horizontal plane. The collimated bean passes through a windowless gas cell where some of the ions are stripped of their extra electron, then into a region containing $2 i \mathrm{l}$ electric field of about $1 \mathrm{kV} / \mathrm{cm}$. There atoms left in the $2 S$ state asquire $2 \mathrm{P}$ state amplitude through the stark effect and quickly decay to the ground state. The resulting Lyman-alpha photon can be detected by special photomultiplier tubes viewing the quench region. The hydrogen atoms next traverse the region between the deflector plates, in which a strong vertical electric field separates the unstripped and doubly stripped components of the beam from the neutral component. The charged components are counted to moritor the relative beam intensity. Finally, the neutral atoms pass through a third set of slits and into a plastic scintillator where they are counted.. The slits are mounted on a translation stage capable of moving in one- $\mu \mathrm{m}$ steps. By 
stepping the final slit in front of the scintillator, the distribution in positior, and hence in ingse, of the neutral beam is measured. A coincidence with a photon indicates that the neutral ator was in the $2 \mathrm{~s}$ state following stripping. The separate components of this apparatus will be described in greater detail below.

\section{b. Beam Collimation}

Techniques for collimating beams to high precision have been developed for the construction of nuclear microprobes, both for negative ${ }^{5}$ and positive ${ }^{6}$ beams. The group at Heidleberg ${ }^{6}$ emphasized careful slit design and fabrication to minimize the number of scattered atoms left in the beam after passing through the slits. They demonstrated the feasibility of collimating a beam to an angular spread of a few microradians with an apparacus a few meters long. The advantage of working with negative ions was demonstrated at Los Alamos, where Armstrong and Wegner ${ }^{5}$ pointed out that the components of the beam that had hit the slit edges would be stripped with high probability and therefore could be separated from the transmitted portion by electrostatic or magnetic deflection of the unstripped beam. It is our further observation that the part of the beam that passes through the edges of the slits is spreed over a rather broad angular range by multiple scattering, so that little of this is mixed with the transmitted beam. We have taken advantage of all of these considerations in the design of our collimator. 
The slits thamselves are

indicated schematically

in Fig. 2. Since most

of the particles hitting

the slit scatter into

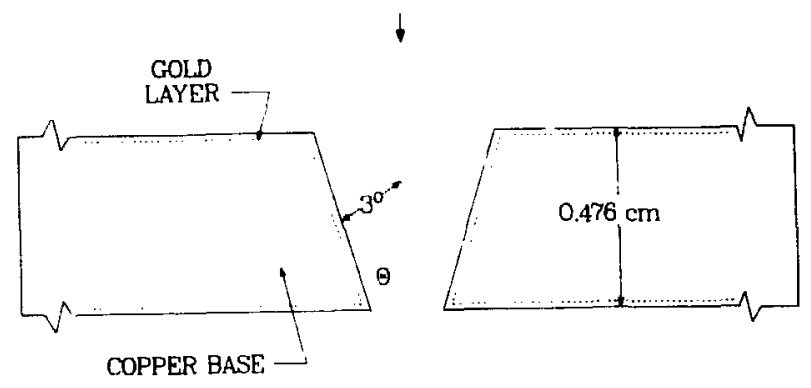

forward angles, it is

desirable that the slit

face be neariy parallel Fig. 2. Diagram of micro-collimating to the beam direction, imately two microns.

so that the beam penetrates only a narrow region of the slit edge. Our slits were made with a $3^{\circ}$ angle. The edges of the slit should be as clean and smooth as possible. The slits were fabricated in the Los Alamos micromachining shop, where flat surfaces with machining marks smaller than $0.03 \mu \mathrm{m}$ are readily achieved. Blanks were first machined frorn copper, then gold was electroplated onto the copper. The two surfaces forming the slit edge were then machined flat, with the tool mo:ing into the edge. The slits were then examined with a scanning electron microscope (SEM) for quality. Some roughness was present on the edges, but in most cases it was well below one $\mu \mathrm{m}$ in size. In practice, these slits worked very well.

The slits were mounted against a flat surface, and the slit width was adjusted with screw driven wedges. The slit opening was measured with the SEM since at a few $\mu \mathrm{m}$ the width of the diffraction pattern with ordinary light is too broad to permit straightforward lise of optical techniques. The second 
collimating slit and the analyzing slit widths were set at about two $\mu \mathrm{m}$. The first collimating slit was found to close when set at two $\mu \mathrm{m}$ and exposed to the beam. When the opening was set at five $\mu \mathrm{m}$, it closed down to around two $\mu \mathrm{m}$, producing a satisfactory beam.

The slits were mounted on a three-meter-long optical table ${ }^{7}$ for vibration suppression. The only coupling of the tablı to the other parts of the apparatus, such as the vacuum system, was through flexible bellows. Mechanical vibration proved to be no problem in the experiment, so further mechanical isolation was not necessary.

The collimating slits were mounted on precision translation stages $^{8}$ identical to those used for the analyzing slit so that they could be moved for alignment. In addition to the microslit, each stage contained a $130-\mu \mathrm{m}$ slit and a circular hole $6.35 \mathrm{~mm}$ in diameter. The slits were first positioner approximately by optically aligning the mounts. Next, the beam was brought through the $6-\mathrm{mm}$ holes, and then the coarse slits were translated to the beam position. Finally the precision slits were brought into position, using the final scintillator to detect the beam.

\section{c. The stripper}

The stripper cell is a cylinder $150 \mathrm{~mm}$ long and about $50 \mathrm{~mm}$ in diameter through which gas can be flowed. The beam enters and leaves the cell through slits $0.4 \mathrm{~mm}$ wide by $2.3 \mathrm{~mm}$ high. The box in which the stripper is mounted is pumped by a $2400-$ 
liter/second diffusion pump, and baffles in the beamline inhibit the migration of gas from this region into other parts of the apparatus. The pressure in the stripper is measured by a baration gauge mounted outside the chamber. It proved necessary to mount the stripper cell on the optical table to preserve alignment when the system was evacuated.

The stripper had to be shielded from magnetic fields; a simple calculation shows that, in a field of 0.5 Gauss perpendicular to the beamline, an ion stripped at the front of the $150-\mathrm{mm}-$ long cell deflects 30 microradians less than one stripped at the back of the cell. Consequently, a high-permeability magnetic shield was placed around the stripper. The field inside this shield, at the entrance and exit of the stripper, was below 0.01 Gauss.

\section{d. The 28 Detector}

The $2 \mathrm{~s}$ component of the beam was identified by a coincidence between a Lyman-alpha photon and a proton in the last scintillator. The photons were detected in UV-sensitive phototubes obtained commercially $y^{9}$. Coincidence rates of one count per second were achieved at the peak of the distribution.

\section{e. The Beam Monitor}

The charged particle beams coming out of the stripper were split in an electric field produced by a parallel plate deflector immediately following the quench plates. The plates are 50-cm long, with a 2-cm separation. Applied fields of 
- $4 \mathrm{kV}$ were sufficient to deflect the charged beams into

plastic scintillators mounted about $0.6 \mathrm{~cm}$ above and below the center of the beamline. The sum of the counts in these counters proved to be a reliabie monitor of the beam current.

\section{f. The Position-sensitive vetector system}

The angular measurements were done by using a precision translation stage to move a fine slit in front of a particle detector. A proton energy spectrum, measured with good resolution, establisied that the microcollimated beam contained only a negligibly small energy degraded component, and that very good energy resolution was not needed in the final detector. consequently, a plastic scintillator like the monitox detectors was used to detect the neutral atoms.

The stage was positioned by a stepper motor under control of a microcomputer. The posj.tion was read by an optical encoder and was recorded as the stage was stepped. The stage moved reliably in one- $\mu \mathrm{m}$ steps.

\section{Data and Analysis}

Figure 3 shows the horizontal profile of the micro-

collimated beam as analyzed by the position detector. The shape is approximately

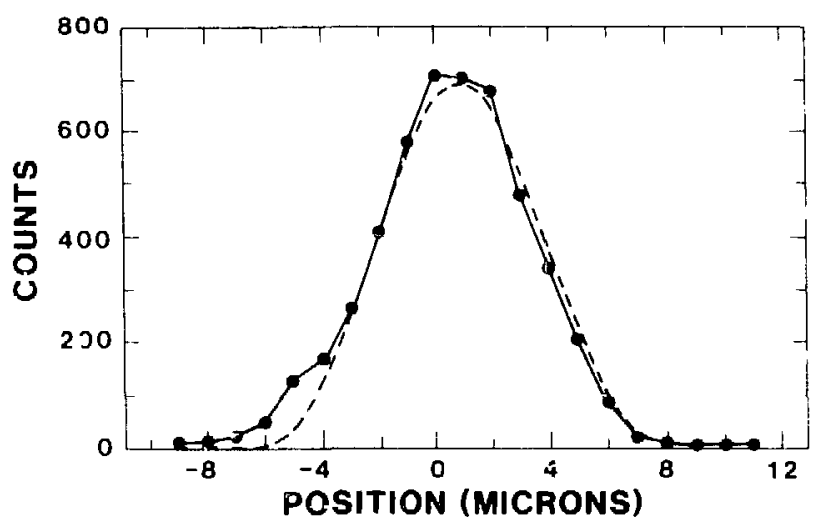
Gaussian and $8 \mu \mathrm{m}$ wide at half maximum. since the distance from the second

Fig. 3. Position scan of the collimated beam. The dashed curve is a Monte Carlo simulation, with 2.5 and $2 \mu \mathrm{m}$ slit widths. 
collimating slit is 2.2 meters, the angular width is about 4 microradians. This was typical of the beam quality we achieved. The dashed curve is the result of a Monte Carlo simulation of the slit system. It is sensitive to the widths of the slits; the dashed curve shown was calculated with the width of the front set at $2.5 \mu \mathrm{m}$ and the two others set at $2 \mu \mathrm{m}$.

In Fig. 42 we show the horizontal profile of a neutral hydrogen beam produced by stripping of $\mathrm{H}^{-}$on nitrogen at about 20 millitorr. The width of the curve is about $30 \mu \mathrm{m}$ FWHM, much larger than the width of the beam from Fig. 3. Figure $4 \mathrm{~b}$ shows the distribution of neutrals measured with

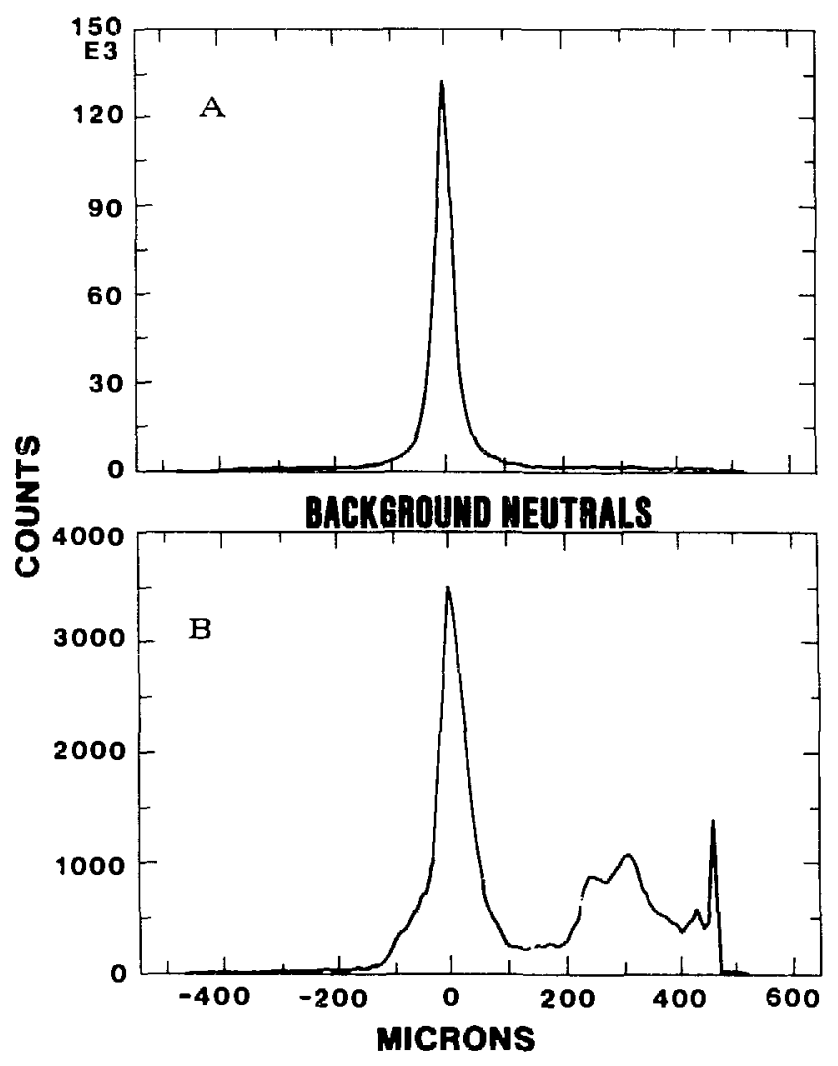
the gas supply to the stripper closed. Because of the bending of the $\mathrm{H}^{-}$beam by the magnetic field of the Earth, the position of Fig. 4a. Singles rate for neutral hydrogen as a function of slit position. The stripping gas was $\mathrm{N}_{2}$ at a pressure of 20 milititorr. The solid curve connects the data points. Fig. 4b. The singles rate with the gas feed closed. The vertical scale is about 40 times smaller than in Fig. 4 a.

the neutral peaks 
depends on where the stripping took place; the peaks in Fig. $4 \mathrm{~b}$ are produced by collisions within various parts $01^{2}$ the beamline. subsequent tuning of the beam eliminated all but a small peak coincident with the peak of Fig. 4a; the small peak was probably produced by collisions with residual gas in the stripper cell.

Figure 5 shows the singles data from stripping on nitrogen with a Lorentzian curve fit to it. (The distance scale has been divided by 1.54 meters, the separation of the

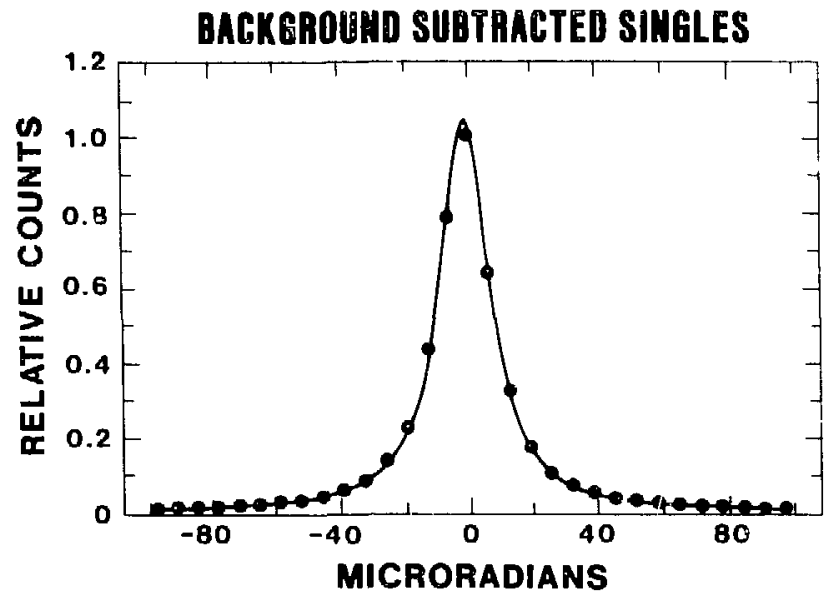
slit and center of the stripper, to give the angle of deflection Fig. 5. Singles data for nitrogen stripper at 20 millitorr. The curve is a Lorentzian fit to the data with 18.4 microradians FWHM.

in urad.) The fit is

clearly very good and suggests that a Lorentzian provides a useful characterization of the data. For the singles data, the FWHM is about 18 microradians.

similar singles data for strippiny on hydrogen gas are shown in Fig. 6. In Fig. 6a the hydrogen pressure was 12.8 millitorr; the width of the best-fit Lorentzian is 19.6 microradians. Figure $6 \mathrm{~b}$ shows data and $a$ fit at 25.4 millitorr. The width of the Lorentzian curve is 
18.7 microradians. It probably is not significant that the width for the $h$ igher gas pressure is slightly smaller; this probably indicates that the sens:tivity of the ficting procedure is limited. We note, however, that the widths are essentially the same as those for the nitrogen data.

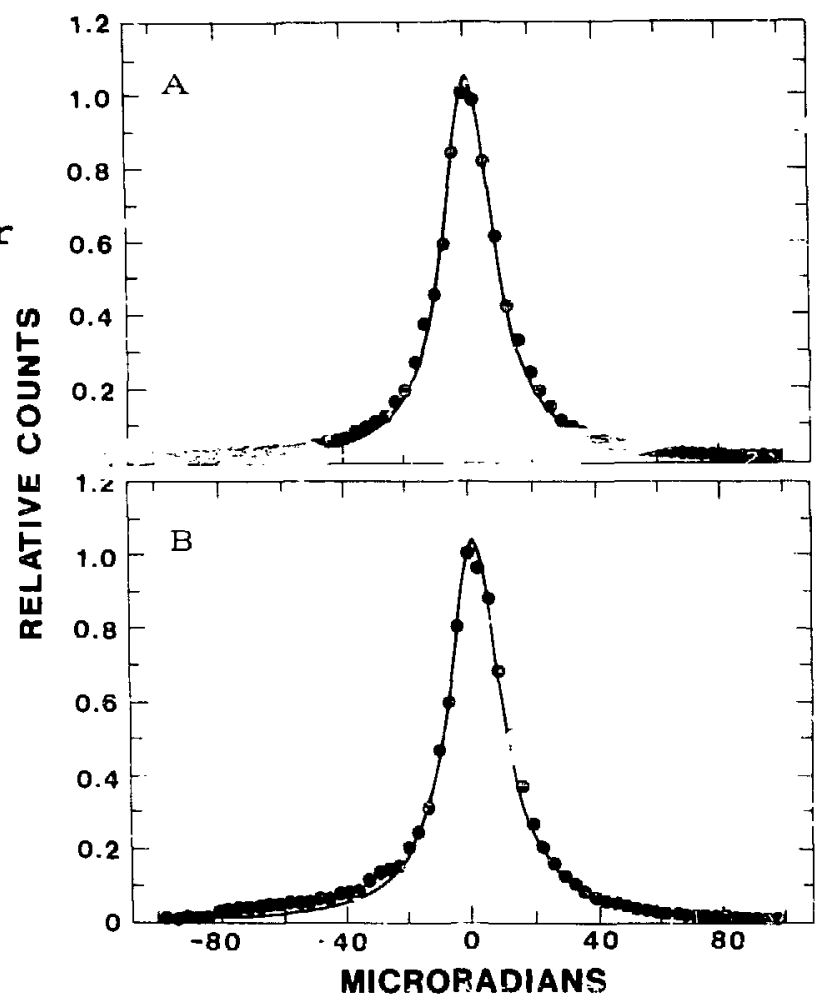

Figure 7 shows the angular distribution of neitrals in coincidence with Lymanalpha photons in the quench region. The stripping gas Fig. 6a. Singles data for hydrogen stripper at 12.8 millitorr. The curve is a Lorentzian fit with 19.6 microradians FWHM.

Fig. 6b. Singles data for hydrogen stripper at 25.4 millitorr. The curve is a Lorentzian fit with 18.7 microradians FWHM was nitrogen at 20 millitorr pressure. The shape is approximately the same as that of the singles data but roughly 2.5 trues wider. The solid curve is a Lorentzian curve of 50 microradians FWHM. As for the singles data, the quality of the fit indicates that one can usefully parameterize the data by a Lorentzian width.

The data presented here aro an average over scattering angle. The height of the slits is very large in comparison to the widith of the distributions of the stripped atoms in both the 
ground and excited states.

Corsequently the measured

intensity at any siit

position is proportional

to the differential cross

section summed over the

range of polar scattering

angles passed by the slit.

The result is a broadening

of the measured distri-

bution zompared to what

one would obtain with

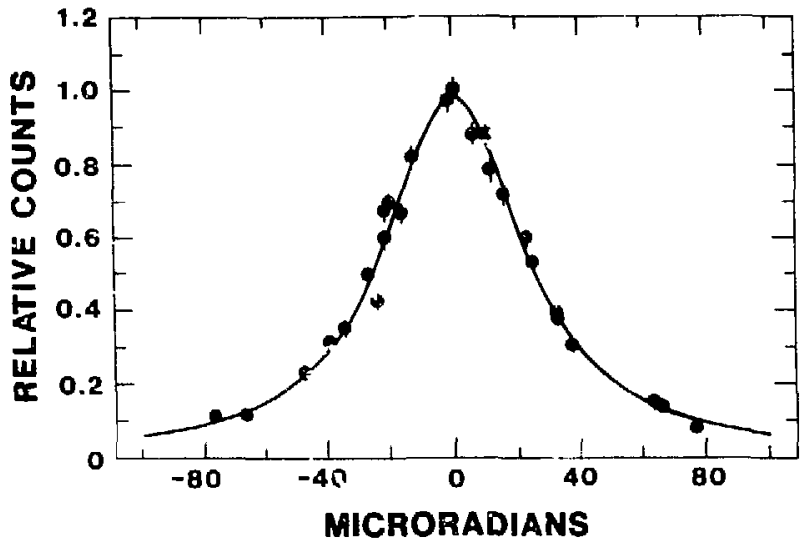

Fig. 7. The 25 coincidence rate as a function of angle. The target gas was nitrogen at 20 millitorr

pressure. The vertical bars indicate the statistical uncertainties. The solid curre is a Lorentzian fit with 50 microradians FWHM.

collimation in two aimensions

and a distortion of its shape. The distribution before averaging is not Lorentzian.

In Fig. 8 we compare the calculations of Wright and Genoni $^{3}$ to the coincidence data. Their calculations used

uncorrelated bound-state wave functions and plane outgoing waves; the theoretical curves have been integrated cver slit

BORE APPAOXIMATION CALCULATIOH FOR THE 2S STATE

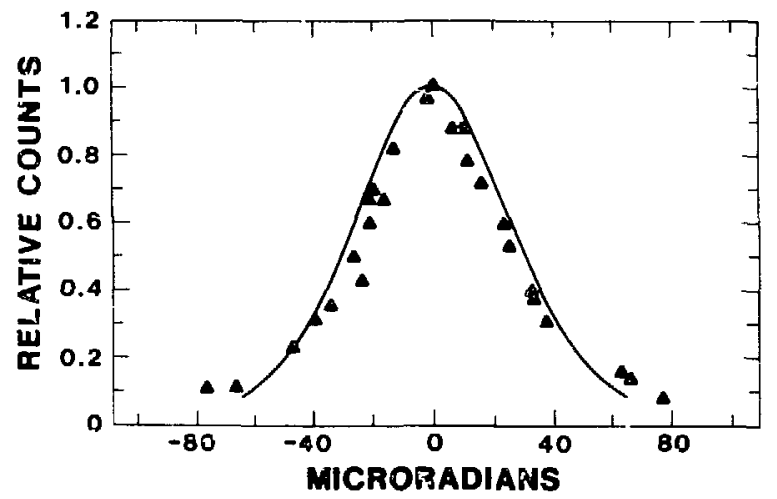

Fig. 3. The averaged Born arproxination curve compared to the coincialence data. Both curves are nomalized to unity at the maximum.

height and normalized to unity at the peak. The data are normalized the same way. The fit is reasonzbly good in the 
central region but deviates significantly from the data in the wings of the distribution.

BACKEBOU:D SUBTAACTEO SIMGLES

The singles data contain contributions from all neutral species produced in the str.pper. The curves in Fig. 9 are weighted averages of the integrated ground state and $2 \mathrm{~s}$ calculations of Wright and Genoni. The points are the singles data of Fig. 4. If we assume that the distribution

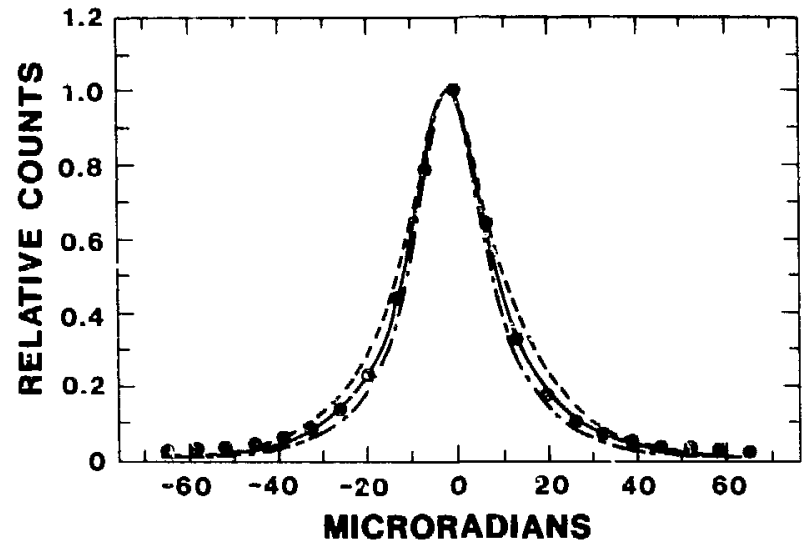

Fig. 9. The singles data fitted by a mixture of sround state and excited state distributions. The solid curve has a $25 \%$ excited state mixture. The dots represent the data. The dashed and dash-dot lines have $35 \%$ and $15 \%$ excited state, respectively.

for hydrogen left in the $2 \mathrm{~S}$ state is representative of all excited-state distributions, this permits us to estimate the fraction of hydrogen left in excited states by the stripping process. The center solid curve, with $25 \%$ of the stripped hydrogen in the excited states, represents the data very well. I'he other curves are for $15 \%$ and $35 \%$ excited-state fractions.

\section{Discussion}

Elased on the comparison of our data with the theory, the stripping of several $\mathrm{MeV} \mathrm{H}^{-}$by gas molecules seems to be reasonably well understood. The angular width of the $2 \mathrm{~s}$ component, if not the full distribution, is reasonably well 
accounted for by calculations using the Born approximation. The measured singles angular distribution is also weli fit by the calculations if aboit $25 \%$ of the observed neutral atoms are assumed to be ieft in excited states by the stripping process. The ridth of the excited state component is -2.5 times greates tisan that of the grounc state component of the stripped beam, as expected, and the effect of the choice of stripping gas on the widths of the angular distributions appears to be vary smail.

More work remains to be done. We should repeat these measiremeats at higher and lower energies to confirm that the widths scale as the square root of the beam energy, as prejicted by the Born approximation. Measurements with a d-beam at one energy vould be useful to test theoretical expectations. Measurements of the populations of specific final states, especially the $2 \mathrm{~S}$, as a function of stripper thickness are important, and might be done with the existing apparatus.

\section{REFERENCES}

1. G. H. Gillespie, Phys. Rev. A 15, 563 (1977).

2. Y. T. Lee and J. C. Y. Chen, Phys. Rev. A 19, 526 (1979).

3. $I$. C. Genoni and L. A. Wright, J. Phys. B 13 , L61 (1980).

4. M. Franz, L. A. Wright, and T. C. Genoni, accepted for publication in Phys. Rev. A.

5. D. D. Armstrong and F. E. Wegner, Rev. Sci. Instr. $\underline{42}, 40$ (1971).

6. R. Noviling, Y. Civelekoglu, B. Povh, D. Schwalm, and K. Traxel, Nucl. Instr. and Meth. 130, 325 (1975).

7. 1983-84 Catalog, Newport Corp., Fountain Valley, CA.

8. Catalog 584, kilinger Scientific, Richmond Hill, N.Y.

9. 1983 Catalog, Hammanatsu Corp., Middlesex, N.J. 